\title{
Fibrinogen performs better than D-dimer for the diagnosis of periprosthetic joint infection: a meta-analysis of diagnostic trials
}

Liping $\mathrm{Pan}^{\dagger}$, Hao Wu${ }^{\dagger}$, Heng Liu, Xin Yang, Zhichao Meng and Yongping Cao ${ }^{*}$ (D)

\begin{abstract}
Purpose: D-dimer and fibrinogen, both belonging to coagulation parameters, are controversial for the diagnosis of periprosthetic joint infection (PJI). This meta-analysis was conducted to compare their diagnostic accuracies for PJI by synthesizing currently available evidence.

Methods: Cochrane Library, MEDLINE, Web of Science, and Embase up to March 1, 2020, and other relevant articles were searched. Five hundred and eighty-one articles were identified after initial research, and 11 studies were included finally. No threshold effects were found between studies. The pooled sensitivity, specificity, and positive and negative likelihood ratio were reported to evaluate the diagnostic performance with heterogeneity analysis. $Z$ test statistics was used to analyze the difference of diagnostic performance between D-dimer and fibrinogen.

Results: The pooled sensitivity, specificity, and positive and negative likelihood ratio of D-dimer for PJI were 0.79 (95\% [CI], 0.72-0.85), $0.77(0.67-0.84), 3.38$ (2.21-5.18), and 0.27 (0.18-0.41), respectively. As for fibrinogen, the pooled sensitivity, specificity, and positive and negative likelihood ratio for PJI were 0.75 (0.68-0.80), 0.85 (0.82-0.88), $5.12(4.22-6.22)$, and $0.30(0.23-0.37)$, respectively. Great heterogeneity was found in studies for D-dimer, and univariate meta-regression analysis revealed that number of involved joints, disease spectrum, comorbidities influencing D-dimer, and sample sources were the source of heterogeneity. $Z$ test found that the pooled specificity of fibrinogen was significantly higher than $\mathrm{D}$-dimer $(0.85 \pm 0.01$ versus $0.77 \pm 0.04, p=0.03)$. The pooled positive likelihood ratio of fibrinogen was significantly higher than D-dimer (5.12 \pm 0.51 versus $3.38 \pm 0.74, p=0.03)$.

Conclusion: Based on currently available evidence, the meta-analysis suggests that fibrinogen performs better than D-dimer as a rule-in diagnostic tool for its higher specificity. However, more prospective trials with larger size are still needed to provide further confirmation.

Trial registration: This meta-analysis was prospectively registered on PROSPERO (International prospective register of systematic reviews), and the registering number was CRD42020177176.
\end{abstract}

Keywords: Periprosthetic joint infection, D-dimer, Fibrinogen, Diagnosis, Meta-analysis

\footnotetext{
* Correspondence: freehorse66@163.com

Pan Liping and Wu Hao are co-first authors

${ }^{\dagger}$ Liping Pan and Hao Wu contributed equally to this work.

Department of Orthopedics, Peking University First Hospital, No. 8 Xishiku

Street, XiCheng District, Beijing 100034, People's Republic of China
}

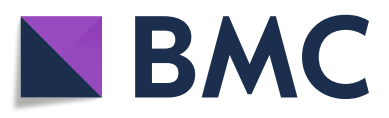

(0) The Author(s). 2021 Open Access This article is licensed under a Creative Commons Attribution 4.0 International License, which permits use, sharing, adaptation, distribution and reproduction in any medium or format, as long as you give appropriate credit to the original author(s) and the source, provide a link to the Creative Commons licence, and indicate if changes were made. The images or other third party material in this article are included in the article's Creative Commons licence, unless indicated otherwise in a credit line to the material. If material is not included in the article's Creative Commons licence and your intended use is not permitted by statutory regulation or exceeds the permitted use, you will need to obtain permission directly from the copyright holder. To view a copy of this licence, visit http://creativecommons.org/licenses/by/4.0/ The Creative Commons Public Domain Dedication waiver (http://creativecommons.org/publicdomain/zero/1.0/) applies to the data made available in this article, unless otherwise stated in a credit line to the data. 


\section{Introduction}

Periprosthetic joint infection (PII) is a rare but devastating complication for patients undergoing joint arthroplasty [1]. It has a huge impact on the joint function and quality of life of patients, causes significant morbidity, and accounts for a substantial proportion of health care expenditure [2]. The Musculoskeletal Infection Society (MSIS) diagnostic criteria [3] for PJI of hips and knees, slightly revised in 2013 by the International Consensus Meeting (ICM) [4], has obtained widespread recognition.

Saxena et al. [5] first discovered an association between coagulopathy and PJI, and some studies extended the research targeting D-dimer and fibrinogen as potential tools for PJI diagnosis. Circulating D-dimer is the characteristic product of degradation of cross-linked fibrin, and fibrinogen is a protein for blood coagulation produced primarily by hepatocytes, both of which could be assessed easily and conveniently in coagulation measurements. Based on the previous research, the new evidence-based 2018 criteria [6] for PJI were published with a better diagnostic accuracy, in which a minor criterion of serum D-dimer was added.

However, some studies [7-17] have demonstrated controversial diagnostic accuracy of D-dimer and fibrinogen as a single biomarker for PJI recently. Some showed that D-dimer was not accurate enough to distinguish PJI and aseptic loosening [8], and others believed fibrinogen could perform better than plasma D-dimer for PJI diagnosis [15], though D-dimer was minor criteria and fibrinogen was not in the new evidence-based 2018 criteria [6] for PJI.

Thus, this meta-analysis aimed to explore the accuracies of D-dimer and fibrinogen for the diagnosis of PJI via synthesizing current available evidence. We sought to determine whether fibrinogen performed better than D-dimer for PJI diagnosis.

\section{Methods and materials \\ Search strategy and criteria}

A systematic approach as required following the Preferred Reporting Items for Systematic Reviews and Meta-Analyses (PRISMA) guidelines [18] was used. A protocol was registered previously online with PROSPERO (International prospective register of systematic reviews), as recommended by PRISMA, and the registration number was CRD42020177176. Cochrane Library, MEDLINE, Web of Science, and Embase from inception up to March 1, 2020, were searched. Keywords or mesh words used were as follows: ("prosthesis-related infections" OR "periprosthetic joint infection" OR "prosthetic infection" OR "joint prosthesis" OR "arthroplasty" OR "replacement") AND "infection" AND ("D-dimer" OR "fibrinogen"). Other relevant articles and their bibliographies were searched in a manually manner after the initial search. It was restricted to publications in English.

\section{Inclusion and exclusion criteria}

The inclusion criteria for studies included patients who underwent joint arthroplasties, confirmed PJI by the MSIS or AAOS guidelines, D-dimer or fibrinogen detected before revision, and sufficient data for sensitivity and specificity. Exclusion criteria included case reports, expert opinions, animal experiments, reviews, unrelated biomarkers, duplications, publications in other languages, and not sufficient data to extract. Two independent reviewers (LP and HW) who were board-certified orthopedic surgeons conducted the screening of all of the studies. Any discrepancy was reported to a third reviewer for a final decision. A total of 581 records were identified after initial screening. One hundred and fiftyfive duplicates were removed, and 415 were excluded after filtering through titles, abstracts, and full texts. Eventually, a total of 11 eligible studies were included in qualitative and quantitative analysis [7-17]. A PRISMA flow diagram of screening studies through this metaanalysis is provided in Fig. 1.

\section{Assessment of study quality}

Totally, 1465 subjects were included in studies for Ddimer, and 1039 subjects were included in studies for fibrinogen. Of these 11 studies, 4 were prospective studies, while the remaining 7 were retrospective. The average age of the subjects ranged from 61.60 to 68.89 years old. Among these 11 studies, 9 studies used Ddimer for PJI diagnosis, 4 used fibrinogen, and 2 evaluated both D-dimer and fibrinogen in the same trial. Except two subjects in one study had other joints involved [7], all other subjects in the studies had only knee and hip arthroplasties involved. The detailed clinical and methodological characteristics are shown in Table 1.

The quality assessment was evaluated using the QUADAS-2 tool [19] as shown in Fig. 2. Two independent reviewers (LP and $\mathrm{HW}$ ) conducted the screening of all of the studies. Any discrepancy was reported to a third reviewer for a final decision. Four studies $[8,10,12,17]$ were considered to have unclear risk of bias in patient selection due to improper patient inclusion criteria in original trials. Two studies $[15,17]$ were considered to have unclear risk of bias in the flow and timing due to not including all consecutive patients.

\section{Data collection and extraction}

Two independent reviewers (LP and HW) extracted the data using standardized spreadsheets and were blinded to the studies' institutions during the extraction of data process. Any discrepancy was reported to a third reviewer for a final decision. The data extracted included first author, publication year, country, study design, gender, size of involved joints, sites of arthroplasty, exclusion criteria, disease spectrum, optimal cutoff value of 


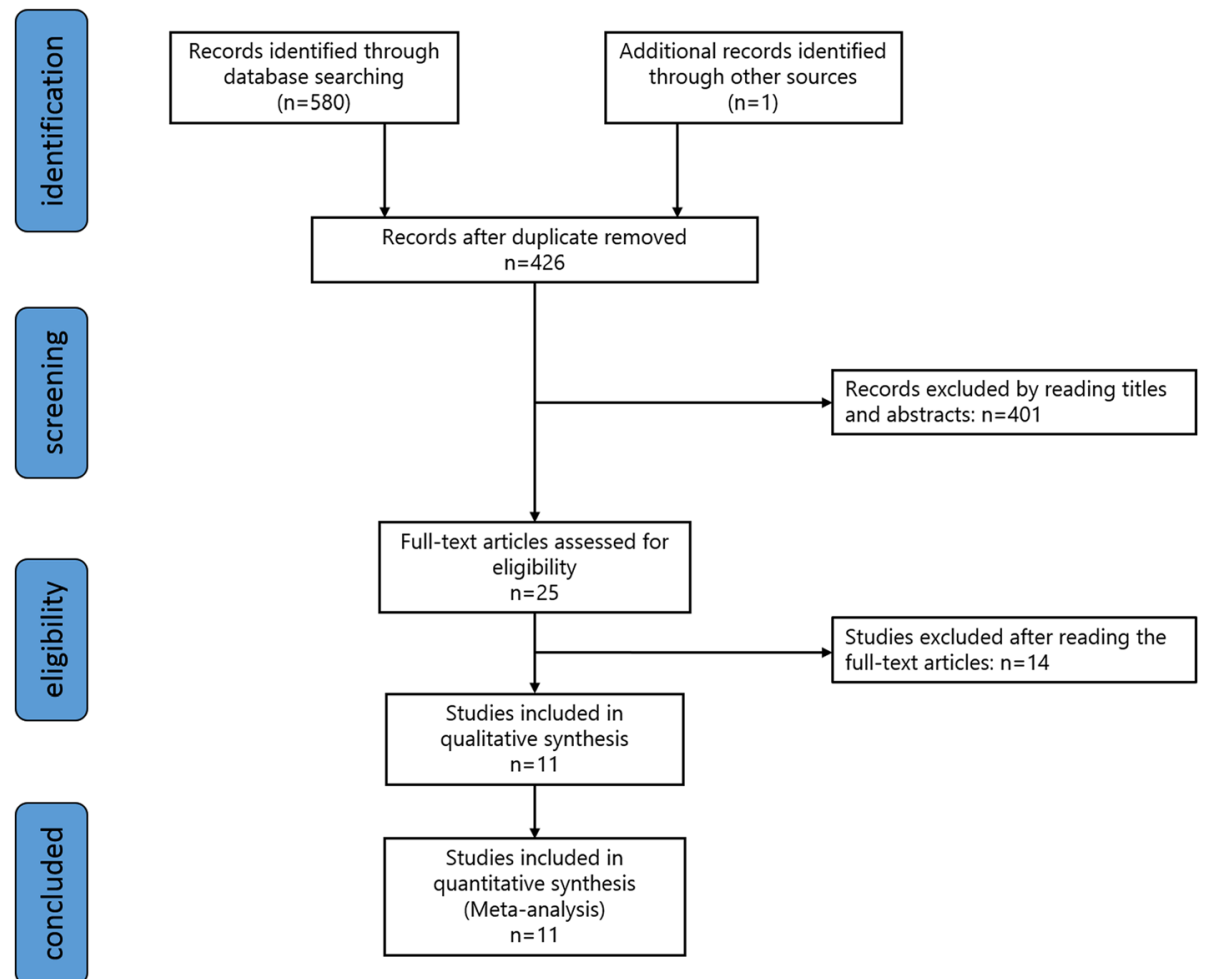

Fig. 1 Flow diagram showing the study screening process

the tests, sensitivity, specificity, or true positive (tp), false positive (fp), false negative (fn), true negative (tn) values if provided, and the referenced gold-standard tests.

\section{Statistical analyses}

Spearman's correlation coefficient was used to assess a possible threshold effect because different studies might have used different cutoff values, which greatly influenced the estimation of sensitivity and specificity. The threshold for D-dimer and fibrinogen was mentioned in all studies, as shown in Table 1. No threshold effect (suggested by a strong positive correlation) was found in studies for D-dimer (Spearman's correlation coefficient - 0.048; $p=0.911$ ) and fibrinogen (Spearman's correlation coefficient $0.40 ; p=0.60$ ).

Without threshold effect, statistics for diagnostic accuracy of D-dimer and fibrinogen for PJI including sensitivity, specificity, positive likelihood ratio $(\mathrm{LR}+)$, and negative likelihood ratio (LR-) were calculated. Heterogeneity was estimated using the $I^{2}$ statistic. $I^{2}$ values between 0 and $50 \%$ indicated minor heterogeneity. Values of $>50 \%$ indicated substantial heterogeneity. Metaregression and subgroup analyses were conducted to identify potential sources of bias, such as country, study design, number of involved joints, disease spectrum, comorbidities influencing D-dimer, sample source, and optimal cutoff value by receiver operating characteristic
(ROC) curve, where substantial heterogeneity was found. The software Stata version 15 and the midas commands were utilized for all analyses. Publication bias was assessed using the methods provided by Deeks et al. [20]. The Deeks funnel plots and regression tests indicated a low possibility of publication bias in studies for D-dimer and fibrinogen $(p=0.21$ and $p=0.68$ for Ddimer and fibrinogen, respectively). The difference of the pooled sensitivity, specificity, LR+, and LR- between Ddimer and fibrinogen was analyzed using $Z$ test statistics, and $p$ value of $<0.05$ was considered significant.

\section{Results}

\section{Diagnostic performance of D-dimer for PJI}

Nine studies [7, 8, 10-15, 17] including 1465 subjects were synthesized for the diagnosis performance of $\mathrm{D}$ dimer for PJI. The pooled sensitivity and specificity of D-dimer for PJI were 0.79 (95\% confidence interval [CI], 0.72 to 0.85 ) and 0.77 ( $95 \% \mathrm{CI}, 0.67$ to 0.84 ), respectively (Fig. 3a). Substantial heterogeneity was identified among studies as the $I^{2}$ values for sensitivity and specificity were 76.08\% (95\% CI, 60.5 to $91.65 \%$ ) and $92.36 \%$ (95\% CI, 88.73 to $95.99 \%)$, respectively. The pooled positive and negative likelihood ratio of D-dimer for PJI were 3.38 (95\% [CI], 2.21 to 5.18 ) and 0.27 (95\%CI, 0.18 to 0.41 ), respectively (Fig. 3b). The $I^{2}$ values for positive and negative likelihood ratio were $89.96 \%$ (95\% CI, 89.96 to 
Table 1 Characteristics of included studies

\begin{tabular}{|c|c|c|c|c|c|c|c|c|c|}
\hline Study & Country & $\begin{array}{l}\text { Study } \\
\text { design }\end{array}$ & $\begin{array}{l}\text { Gender } \\
\text { (F/M) }\end{array}$ & $\begin{array}{l}\text { Age (years } \\
\pm \text { SD) }\end{array}$ & $\begin{array}{l}\text { Involved } \\
\text { joints } \\
\text { (PJI/total) }\end{array}$ & $\begin{array}{l}\text { Sites of } \\
\text { arthroplasty }\end{array}$ & Disease spectrum & Comorbidities * & Cutoff \\
\hline \multicolumn{10}{|l|}{ D-dimer } \\
\hline Qin et al. [11] & China & $P$ & $69 / 53$ & $65.21 \pm 10.51$ & $55 / 122$ & Hips and knees & Chronic PJI + aseptic & Excluded & $1170 \mathrm{ng} / \mathrm{ml}$ \\
\hline Xiong et al. [13] & China & $P$ & $48 / 32$ & $61.60 \pm 12.22$ & $26 / 80$ & Hips and knees & PJI + aseptic & Excluded & 756 ng/ml \\
\hline Shahi et al. [12] & USA & $P$ & $94 / 101$ & 62.46 & $57 / 195$ & Hips and knees & $\begin{array}{l}\text { Primary arthroplasty } \\
+\mathrm{PJ}+\text { aseptic }+ \\
\text { reimplantation }\end{array}$ & Not excluded & $850 \mathrm{ng} / \mathrm{mL}$ \\
\hline Xu et al. [14] & China & $\mathrm{R}$ & NM & NM & $82 / 224$ & Hips and knees & PJI + aseptic & Excluded & $1020 \mathrm{ng} / \mathrm{mL}$ \\
\hline Huang et al. [8] & China & $\mathrm{R}$ & $46 / 34$ & $67.15 \pm 2.64$ & $31 / 101$ & Hips and knees & $\begin{array}{l}\text { Primary arthroplasty } \\
+ \text { PJI + aseptic }\end{array}$ & Excluded & $850 \mathrm{ng} / \mathrm{mL}$ \\
\hline Pannu et al. [10] & USA & $\mathrm{R}$ & $62 / 49$ & $68.89 \pm 10$ & $49 / 111$ & Hips and knees & PJI + aseptic & NM & $2300 \mathrm{ng} / \mathrm{mL}$ \\
\hline Hu et al. [7] & China & $\mathrm{R}$ & $41 / 36$ & 63.08 & $40 / 77$ & $\begin{array}{l}\text { Hips and knees, } \\
2 \text { others }\end{array}$ & PJI + aseptic & Not excluded & $955 \mathrm{ng} / \mathrm{ml}$ \\
\hline Li et al. [15] & China & $\mathrm{R}$ & NM & NM & $76 / 439$ & Hips and knees & PJI + aseptic & Excluded & $1250 \mathrm{ng} / \mathrm{ml}$ \\
\hline Wu et al. [17] & China & $\mathrm{R}$ & $82 / 54$ & $66.32 \pm 11.73$ & $35 / 116$ & Hips and knees & $\begin{array}{l}\mathrm{PJI}+\text { aseptic+ } \\
\text { reimplantation }\end{array}$ & Not excluded & $410 \mathrm{ng} / \mathrm{ml}$ \\
\hline \multicolumn{10}{|l|}{ Fibrinogen } \\
\hline Li et al. [15] & China & $\mathrm{R}$ & NM & NM & $76 / 439$ & Hips and knees & PJI + aseptic & Excluded & $4.01 \mathrm{~g} / \mathrm{L}$ \\
\hline Wu et al. [17] & China & $\mathrm{R}$ & $82 / 54$ & $66.32 \pm 11.73$ & $35 / 116$ & Hips and knees & $\begin{array}{l}\text { PJI + aseptic+ } \\
\text { reimplantation }\end{array}$ & Not excluded & $3.61 \mathrm{~g} / \mathrm{L}$ \\
\hline Klim et al. [16] & Austria & $P$ & NM & $65.50 \pm 15.31$ & $78 / 124$ & Hips and knees & PJI + aseptic & Excluded & $5.19 \mathrm{~g} / \mathrm{L}$ \\
\hline Xu et al. [9] & China & $\mathrm{R}$ & NM & NM & $153 / 360$ & Hips and knees & PJI + aseptic & Excluded & $3.57 \mathrm{~g} / \mathrm{L}$ \\
\hline
\end{tabular}

$N M$ not mentioned, $P J l$ periprosthetic joint infection, $P$ perspective, $R$ retrospective

*Comorbidities referred to comorbidities influencing D-dimer or fibrinogen

96.29\%) and $86.82 \%$ (95\% CI, 79.50 to $94.15 \%)$, respectively.

\section{Diagnostic performance of fibrinogen for PJ}

Four studies [9, 15-17] including 1039 subjects were synthesized for the diagnosis performance of fibrinogen for PJI. The pooled sensitivity and specificity of fibrinogen for PJI were 0.75 (95\% [CI], 0.68 to 0.80$)$ and $0.85(95 \% \mathrm{CI}, 0.82$ to 0.88$)$, respectively (Fig. $4 \mathrm{a})$. Minor heterogeneity was identified among studies as the $I^{2}$ values for sensitivity and specificity were $33.95 \%$ (95\% CI, 0 to 100\%) and 14.63\% (95\% CI, 0 to 100\%), respectively. The pooled positive and negative likelihood ratio of fibrinogen for PJI were 5.12 (95\% [CI], 4.22 to 6.22) and 0.30 (95\% CI, 0.23 to 0.37 ), respectively (Fig. $4 \mathrm{~b}$ ). The $I^{2}$ values for positive and negative likelihood ratio were $0 \%$ (95\% CI, 0 to 100\%) and 5.75\% (95\% CI, $0 \%$ to $100 \%)$, respectively.

\section{Meta-regression and subgroup analysis}

Heterogeneity was identified for pooled sensitivity and specificity among studies of D-dimer and fibrinogen. Substantial heterogeneity was found in studies for Ddimer, and minor heterogeneity for fibrinogen. Therefore, univariate meta-regression and subgroup analysis were performed to identify potential sources of heterogeneity among studies for D-dimer. The results of the meta-regression and subgroup analysis are shown in Table 2. The significant sources of heterogeneity in terms of sensitivity were number of involved joints, comorbidities influencing D-dimer, and sample source. The significant sources of heterogeneity in terms of specificity were disease spectrum, number of involved joints, and comorbidities influencing D-dimer. Other factors, like country, study design, and optimal cutoff values were not significantly different.

\section{The difference of pooled diagnostic performance between $\mathrm{D}$-dimer and fibrinogen}

$Z$ test statistics was conducted to analyze the difference of the pooled diagnostic performance between D-dimer and fibrinogen, as shown in Table 3. The pooled sensitivity of D-dimer and fibrinogen was $0.79 \pm 0.04$ (value \pm standard error) and $0.75 \pm 0.03$, and no significant difference was found as $p$ value was 0.15 . The pooled specificity of D-dimer and fibrinogen was $0.77 \pm 0.04$ and $0.85 \pm 0.01$, and significant difference was found for $p$ value less than 0.05 . The pooled positive likelihood ratio of D-dimer and fibrinogen was $3.38 \pm 0.74$ and $5.12 \pm$ 0.51 , and significant difference was found for $p$ value less than 0.05 . The pooled negative likelihood ratio of $\mathrm{D}$ dimer and fibrinogen was $0.27 \pm 0.06$ and $0.30 \pm 0.04$, 

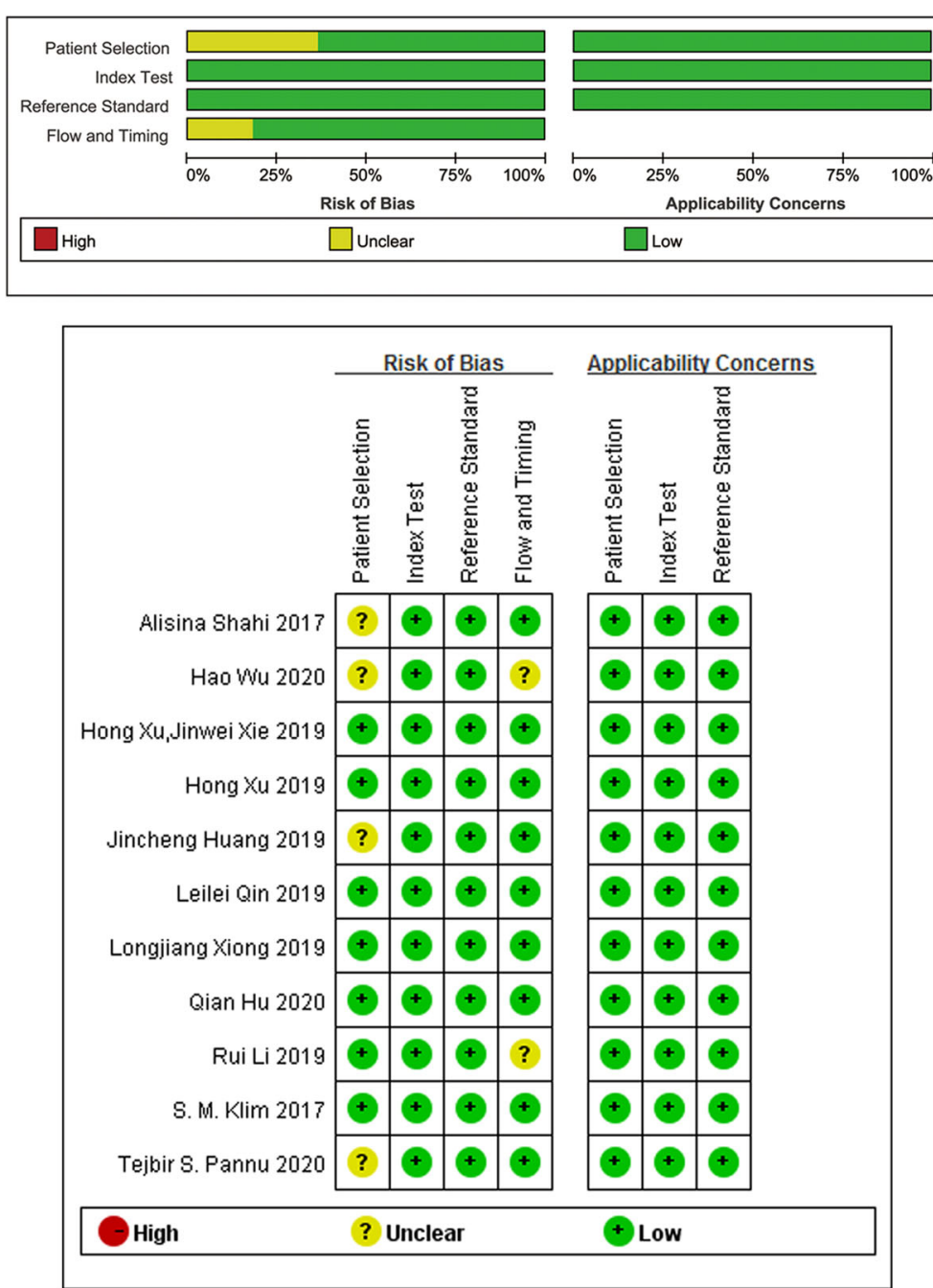

Fig. 2 Grouped bar charts showing the risk of bias and applicability concerns for the 11 included studies according to the QUADAS-2 tool

and no significant difference was found as $p$ value was 0.34 .

\section{Discussion}

Timely and accurate diagnosis of PJI of the hips and knees remains a major challenge for orthopedic surgeons as there are no absolutely accurate tests currently [21, 22]. Numerous biomarkers have been discovered and become available in the recent years, including circulating Ddimer and fibrinogen in coagulation system. D-dimer and fibrinogen could be detected in coagulation test which was essential preoperative procedures. Thus, no additional costs would be incurred if D-dimer and fibrinogen were taken as regular diagnostic tools. This may be an incentive for clinicians to use them more routinely. This metaanalysis aimed to compare the diagnostic accuracy of Ddimer and fibrinogen from the available published studies. Our pooled results showed that D-dimer and fibrinogen performed well in sensitivity and specificity in diagnosing
PJI, and fibrinogen achieved a significantly higher specificity. Fibrinogen had a higher positive likelihood ratio to be qualified as a rule-in diagnostic tool. Both biomarkers had a low negative likelihood ratio, making them suitable to be qualified as rule-out tools. Overall, as a single test, both of them could be used for PJI diagnosis, while fibrinogen had a higher diagnostic value.

This study had a number of limitations. First, not all of the sample sizes were big enough, and most of the studies came from the same region, and were not prospective. These may affect the quality of the trials and might have caused additional bias. Second, although low possibility of publication bias $(p>0.05)$ was observed by Deeks' funnel plots, there was still some extent of publication bias because positive results tend to be more likely to be published. Third, though D-dimer and fibrinogen could be used for PJI diagnosis, the optimal cutoff values were not identified, which was of great importance for clinical applications and could not be 

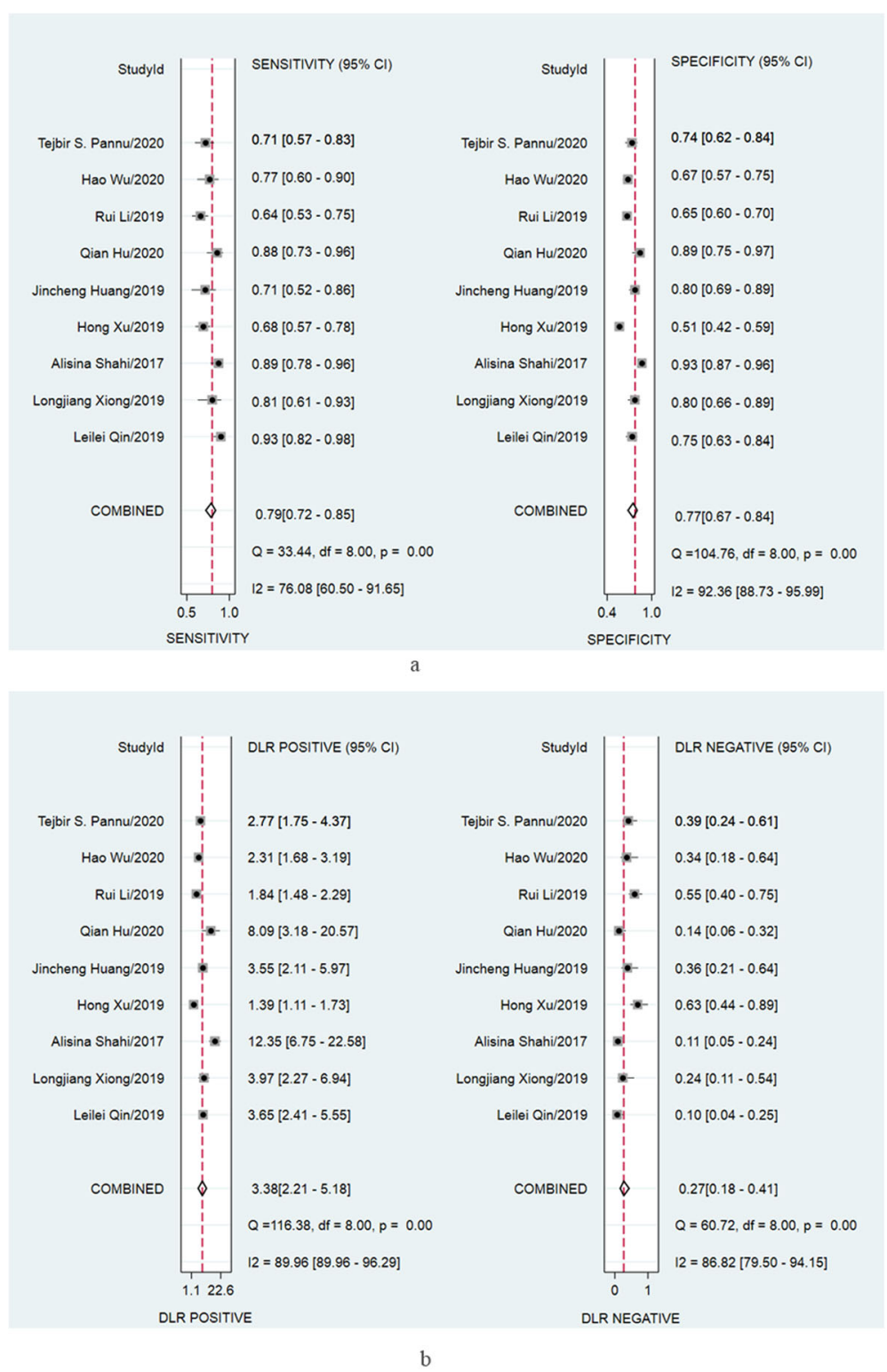

Fig. 3 Paired forest plots of the sensitivity and specificity (a) and positive and negative likelihood ratio (b) of D-dimer for the diagnosis of PJI

resolved by meta-analysis. Fourth, though D-dimer and fibrinogen were considered as good individual biomarkers for PJI, combined tests of D-dimer or fibrinogen with other biomarkers like ESR or CRP might perform even better, which was to be confirmed through further prospective trials.

Both D-dimer and fibrinogen showed high sensitivity and specificity, which indicated that both could be used for clinic practice for PJI diagnosis. A guideline defines that $\mathrm{LR}+>2, \mathrm{LR}-<0.5$ is considered a viable predictor, and $\mathrm{LR}+>5$, LR $-<0.2$ is considered a good predictor [23]. Great heterogeneity was found in studies for Ddimer, and univariate meta-regression analysis revealed that number of involved joints, disease spectrum, comorbidities influencing D-dimer, and sample sources were the source of heterogeneity. As for studies for fibrinogen, minor heterogeneity was found.

D-dimer is a characteristic degradation product of cross-linked fibrin [24]. It is an established screening test for thrombotic diseases and extended its unconventional use to inflammation and infection, especially to PJI. It was proved that D-dimer had a significant differentiating ability in sepsis-related mortality [25]. D-dimer had been considered as one of the minor criteria for its diagnostic accuracy in the newly established evidence-based PJI definition [6]. Although Xu et al. [14] and Huang et al. [8] demonstrated that D-dimer had limited value and was not suitable for PJI diagnosis, our pooled results 

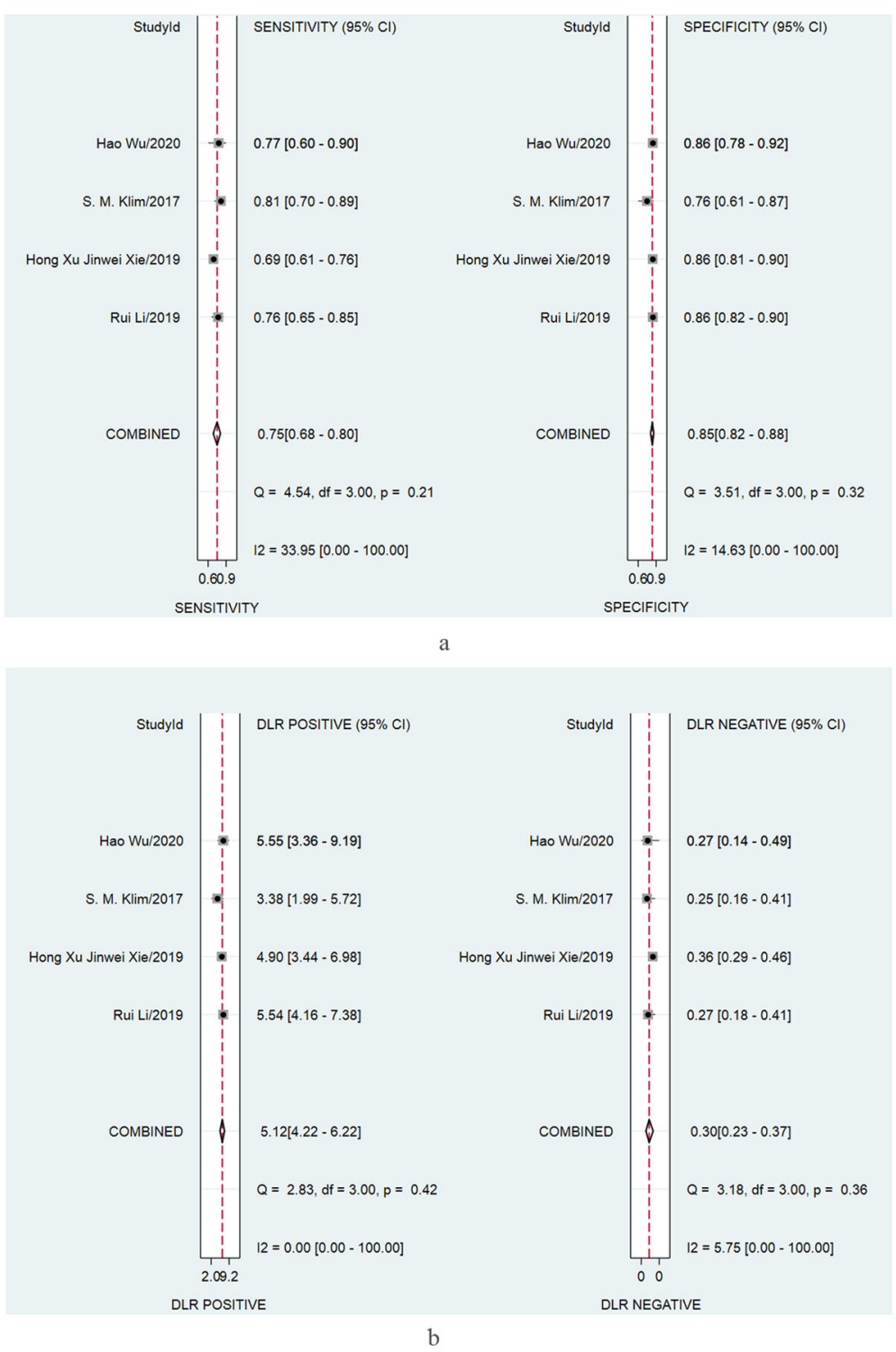

Fig. 4 Paired forest plots of the sensitivity and specificity (a) and positive and negative likelihood ratio (b) of fibrinogen for the diagnosis of PJI

embodied these two studies showed that D-dimer had a high sensitivity and specificity to be a diagnostic tool for PJI, which were consistent with the established criteria.

Fibrinogen, a protein for blood coagulation, is synthesized by hepatocytes [26]. While its key role in the coagulation cascade is general knowledge, fibrinogen also plays a pivotal role in regulating inflammation process and preventing infection [27]. Our results showed that fibrinogen had a high sensitivity and specificity for PJI, which was consistent with the results exploring fibrinogen and D-dimer for PJI in the same trial $[15,17]$. Thus, it could also be used as a biomarker for PJI. Many studies exploring the connection between fibrinogen and infection found reduced fibrinogen lead to compromised pathogen clearance, exacerbated pathogen infection, and increased mortality following subcutaneous infection $[28,29]$. The underlying mechanism for connection between fibrinogen and PJI is still to be explored.

Substantial heterogeneity was found in studies for D-dimer, and its potential sources were explored by performing meta-regression and subgroup analysis. The results showed that number of involved joints, disease spectrum, comorbidities influencing D-dimer, and sample source were sources of heterogeneity. Comorbidities like inflammatory arthritis, deep vein thrombosis, a prosthetic heart valve, a history of 
Table 2 Univariable meta-regression and subgroup analysis for potential heterogeneity in studies for D-dimer

\begin{tabular}{|c|c|c|c|c|c|}
\hline Covariate & No. of studies & Sensitivity $(95 \% \mathrm{Cl})$ & $P$ value & Specificity $(95 \% \mathrm{Cl})$ & $P$ value \\
\hline \multicolumn{6}{|l|}{ Country } \\
\hline USA & 2 & $0.82[0.69-0.95]$ & 0.27 & $0.86[0.75-0.97]$ & 0.90 \\
\hline China & 7 & $0.79[0.71-0.87]$ & . & $0.73[0.64-0.82]$ & . \\
\hline \multicolumn{6}{|l|}{ Study design } \\
\hline Prospective & 3 & $0.89[0.83-0.95]$ & 0.41 & $0.84[0.75-0.94]$ & 0.71 \\
\hline Retrospective & 6 & $0.73[0.67-0.79]$ & . & $0.72[0.61-0.82]$ & \\
\hline \multicolumn{6}{|l|}{ No. of involved joints } \\
\hline$>150$ & 3 & $0.76[0.64-0.87]$ & $0.01^{*}$ & $0.74[0.58-0.89]$ & 0.14 \\
\hline$<150$ & 6 & $0.81[0.74-0.89]$ & & $0.78[0.68-0.88]$ & . \\
\hline \multicolumn{6}{|l|}{ Disease spectrum } \\
\hline PJI + aseptic & 6 & $0.79[0.70-0.87]$ & 0.06 & $0.73[0.62-0.84]$ & $0.03^{*}$ \\
\hline PJI + aseptic + primary arthroplasty/reimplantaion & 3 & $0.81[0.69-0.92]$ & . & $0.82[0.71-0.93]$ & . \\
\hline \multicolumn{6}{|l|}{ Comorbidities influencing D-dimer } \\
\hline Excluded & 5 & $0.77[0.67-0.86]$ & $0.01^{*}$ & $0.71[0.59-0.82]$ & $0.01^{*}$ \\
\hline Not excluded & 4 & $0.82[0.73-0.91]$ & . & $0.83[0.73-0.92]$ & . \\
\hline \multicolumn{6}{|l|}{ Sample source } \\
\hline Plasma & 4 & $0.75[0.65-0.85]$ & $0.00^{*}$ & $0.68[0.56-0.81]$ & $0.00^{*}$ \\
\hline Serum & 5 & $0.83[0.75-0.90]$ & & $0.82[0.74-0.90]$ & . \\
\hline \multicolumn{6}{|l|}{ Optimal cutoff value } \\
\hline$<900 \mathrm{ng} / \mathrm{ml}$ & 4 & $0.81[0.71-0.91]$ & 0.14 & $0.81[0.72-0.91]$ & 0.54 \\
\hline$>900$ ng/ml & 5 & $0.78[0.69-0.87]$ & . & $0.72[0.60-0.83]$ & \\
\hline
\end{tabular}

*Statistical significance $(p<0.05)$

hypercoagulation disorder, or other infectious diseases would influence the level of D-dimer [11]. The results found higher sensitivity and specificity would be achieved when all these comorbidities were excluded. In order to get a more accurate diagnosis, these comorbidities should be carefully assessed. The metaregression results revealed that serum $\mathrm{D}$-dimer performed better than plasma levels in the diagnosis of PJI. Serum D-dimer was measured after blood clotting with consumption of fibrinogen and some other coagulation factors, which would be different from plasma D-dimer levels. Previous studies did not have a uniform conclusion about the relationship between plasma and serum D-dimer [30-32]. More studies could be conducted to further exploring the difference.

$Z$ test statistics found the pooled specificity and LR+ of fibrinogen were significantly higher than D-dimer, although no significant difference of sensitivity and LRwas found between fibrinogen and D-dimer. These indicated that fibrinogen performed better than D-dimer for PJI diagnosis. These results were consistent with other studies exploring the difference of D-dimer and fibrinogen in PJI diagnosis $[15,17]$. As we know, D-dimer is included as one minor criterion in the newly established diagnosis criteria of PJI in 2018 [6]. Fibrinogen could be

Table 3 Comparison of pooled diagnostic performance of D-dimer and fibrinogen by Z-test statistics

\begin{tabular}{|c|c|c|c|c|c|c|c|c|}
\hline & \multicolumn{3}{|l|}{ D-dimer } & \multicolumn{3}{|l|}{ Fibrinogen } & \multirow[t]{2}{*}{$Z$ value } & \multirow[t]{2}{*}{$p$ value } \\
\hline & Pooled value & SE & $95 \% \mathrm{Cl}$ & Pooled value & SE & $95 \% \mathrm{Cl}$ & & \\
\hline Sensitivity & 0.79 & 0.04 & $0.72-0.85$ & 0.75 & 0.03 & $0.68-0.80$ & 1.02 & 0.15 \\
\hline Specificity & 0.77 & 0.04 & $0.67-0.84$ & 0.85 & 0.01 & $0.82-0.88$ & 1.93 & $0.03^{*}$ \\
\hline LR+ & 3.38 & 0.74 & $2.21-5.18$ & 5.12 & 0.51 & $4.22-6.22$ & 1.94 & $0.03^{*}$ \\
\hline LR- & 0.27 & 0.06 & $0.18-0.41$ & 0.30 & 0.04 & $0.23-0.37$ & 0.42 & 0.34 \\
\hline
\end{tabular}

$S E$ standard error, $C l$ confidence interval, $L R+$ positive likelihood ratio, $L R$ - negative likelihood ratio

${ }^{*} p$ value less than 0.05 meant significant difference 
another better criterion for PJI diagnosis, and more prospective and larger-sized trials should be conducted to confirm it.

\section{Conclusion}

Based on currently available evidence, the meta-analysis suggests that fibrinogen performs better than D-dimer as a rule-in diagnostic tool for its higher specificity. However, more prospective trials with larger size are still needed to provide further confirmation.

\section{Abbreviations}

PJI: Periprosthetic joint infection; MSIS: The Musculoskeletal Infection Society; ICM: International Consensus Meeting; PRISMA: The Preferred Reporting Items for Systematic Reviews and Meta-Analyses; LR+: Positive likelihood ratio; LR-: Negative likelihood ratio; ROC: Receiver operating characteristic

\section{Acknowledgements}

Not applicable.

\section{Authors' contributions}

All authors contributed to the study conception and design. Material preparation, data collection, and analysis were performed by Liping Pan, Hao Wu, Heng Liu, Xin Yang, Zhichao Meng, and Yongping Cao. The first draft of the manuscript was written by Liping Pan and Hao Wu, and all authors commented on previous versions of the manuscript. All authors read and approved the final manuscript.

\section{Funding}

This work was supported by the Natural Science Foundation of Beijing Municipality (NO.7192204)

\section{Availability of data and materials}

All data and materials are contained within the manuscript.

\section{Ethics approval and consent to participate}

No Ethical Review Committee Statement is required, since our study is a meta-analysis.

\section{Consent for publication}

Not applicable.

\section{Competing interests}

The authors declare that they have no competing interests.

Received: 25 October 2020 Accepted: 19 November 2020

Published online: 09 January 2021

\section{References}

1. Huotari K, Peltola M, Jamsen E. The incidence of late prosthetic joint infections: a registry-based study of 112,708 primary hip and knee replacements. Acta Orthop. 2015;86(3):321-5

2. Helwig P, Morlock J, Oberst M, Hauschild O, Hubner J, Borde J, Sudkamp $N P$, Konstantinidis L. Periprosthetic joint infection--effect on quality of life. Int Orthop. 2014:38(5):1077-81.

3. Parvizi J, Zmistowski B, Berbari EF, Bauer TW, Springer BD, Della Valle CJ, Garvin KL, Mont MA, Wongworawat MD, Zalavras CG. New definition for periprosthetic joint infection: from the Workgroup of the Musculoskeletal Infection Society. Clin Orthop Relat Res. 2011;469(11):2992-4.

4. Parvizi J, Gehrke T, Chen AF. Proceedings of the International Consensus on Periprosthetic Joint Infection. Bone Joint J. 2013;95-B(11):1450-2.

5. Saxena A, Baratz M, Austin MS, Purtill JJ, Parvizi J. Periprosthetic joint infection can cause abnormal systemic coagulation. J Arthroplasty. 2011; 26(1):50-7 7 e1.

6. Parvizi J, Tan TL, Goswami K, Higuera C, Della Valle C, Chen AF, Shohat N. The 2018 definition of periprosthetic hip and knee infection: an evidencebased and validated criteria. J Arthroplasty. 2018;33(5):1309-14 e2.
7. Hu Q, Fu Y, Tang L. Serum D-dimer as a diagnostic index of PJI and retrospective analysis of etiology in patients with PJI. Clin Chim Acta. 2020; 506:67-71.

8. Huang J, Zhang Y, Wang Z, Dong Y, Zhao Y, Zheng J, Lian H, Jin Y. The serum level of D-Dimer is not suitable for distinguishing between prosthetic joint infection and aseptic loosening. J Orthop Surg Res. 2019;14(1):407.

9. Xu H, Xie J, Yang J, Chen G, Huang Q, Pei F. Plasma fibrinogen and platelet count are referable tools for diagnosing periprosthetic joint infection: a single-center retrospective cohort study. J Arthroplasty. 2020;35(5):1361-7.

10. Pannu TS, Villa JM, Patel PD, Riesgo AM, Barsoum WK, Higuera CA. The Utility of Serum d-Dimer for the Diagnosis of Periprosthetic Joint Infection in Revision Total Hip and Knee Arthroplasty. J Arthroplasty. 2020;35(6):169295.

11. Qin L, Li F, Gong X, Wang J, Huang W, Hu N. Combined measurement of Ddimer and $\mathrm{C}$-reactive protein levels: highly accurate for diagnosing chronic periprosthetic joint infection. J Arthroplasty. 2020;35(1):229-34.

12. Shahi A, Kheir MM, Tarabichi M, Hosseinzadeh HRS, Tan TL, Parvizi J. Serum D-dimer test is promising for the diagnosis of periprosthetic joint infection and timing of reimplantation. J Bone Joint Surg Am. 2017:99(17):1419-27.

13. Xiong L, Li S, Dai M. Comparison of D-dimer with CRP and ESR for diagnosis of periprosthetic joint infection. J Orthop Surg Res. 2019;14(1):240.

14. Xu H, Xie J, Huang Q, Lei Y, Zhang S, Pei F. Plasma fibrin degradation product and D-dimer are of limited value for diagnosing periprosthetic joint infection. J Arthroplasty. 2019;34(10):2454-60.

15. Li R, Shao HY, Hao LB, Yu BZ, Qu PF, Zhou YX, Chen JY. Plasma fibrinogen exhibits better performance than plasma D-dimer in the diagnosis of periprosthetic joint infection: a multicenter retrospective study. J Bone Joint Surg Am. 2019;101(7):613-9.

16. Klim SM, Amerstorfer F, Gruber G, Bernhardt GA, Radl R, Leitner L, Leithner A, Glehr M. Fibrinogen - a practical and cost efficient biomarker for detecting periprosthetic joint infection. Sci Rep. 2018;8(1):8802.

17. Wu H, Meng Z, Pan L, Liu H, Yang X, Yongping C. Plasma Fibrinogen Performs Better Than Plasma d-Dimer and Fibrin Degradation Product in the Diagnosis of Periprosthetic Joint Infection and Determination of Reimplantation Timing. J Arthroplasty.2020;35(8):2230-6.

18. Moher D, Liberati A, Tetzlaff J, Altman DG, Group P. Preferred reporting items for systematic reviews and meta-analyses: the PRISMA statement. J Clin Epidemiol. 2009;62(10):1006-12.

19. Whiting PF, Rutjes AW, Westwood ME, Mallett S, Deeks JJ, Reitsma JB, Leeflang MM, Sterne JA, Bossuyt PM. QUADAS-2: a revised tool for the quality assessment of diagnostic accuracy studies. Ann Intern Med. 2011; 155(8):529-36.

20. Deeks JJ, Macaskill P, Irwig L. The performance of tests of publication bias and other sample size effects in systematic reviews of diagnostic test accuracy was assessed. J Clin Epidemiol. 2005;58(9):882-93.

21. Fernández-Sampedro M, Fariñas-Alvarez C, Garces-Zarzalejo C, AlonsoAguirre MA, Salas-Venero C, Martínez-Martínez L, Fariñas MC. Accuracy of different diagnostic tests for early, delayed and late prosthetic joint infection. BMC Infect Dis. 2017;17(1):592.

22. Ahmad SS, Shaker A, Saffarini M, Chen AF, Hirschmann MT, Kohl S. Accuracy of diagnostic tests for prosthetic joint infection: a systematic review. Knee Surg Sports Traumatol Arthrosc. 2016;24(10):3064-74.

23. Jaeschke R, Guyatt GH, Sackett DL, Guyatt G, Wilson M. Users' Guides to the Medical Literature: III. How to Use an Article About a Diagnostic Test B. What Are the Results and Will They Help Me in Caring for My Patients? J Am Med Assoc. 1994;271(9):703-7.

24. Adam SS, Key NS, Greenberg CS. D-dimer antigen: current concepts and future prospects. Blood. 2009;113(13):2878-87.

25. Rodelo JR, De la Rosa G, Valencia ML, Ospina S, Arango CM, Gómez Cl, García A, Nuñez E, Jaimes FA. D-dimer is a significant prognostic factor in patients with suspected infection and sepsis. Am J Emerg Med. 2012;30(9): 1991-9.

26. Jennewein C, Tran N, Paulus P, Ellinghaus P, Eble JA, Zacharowski K. Novel aspects of fibrin(ogen) fragments during inflammation. Mol Med. 2011;17(5-6):568-73.

27. Luyendyk JP, Schoenecker JG, Flick MJ. The multifaceted role of fibrinogen in tissue injury and inflammation. Blood. 2019;133(6):511-20.

28. Sun $H$, Wang $X$, Degen $J$, Ginsburg D. Reduced thrombin generation increases host susceptibility to group A streptococcal infection. Blood. 2009; 113(6):1358-64.

29. Prasad JM, Gorkun OV, Raghu H, Thornton S, Mullins ES, Palumbo JS, Ko YP, Höök M, David T, Coughlin SR, Degen JL, Flick MJ. Mice expressing a 
mutant form of fibrinogen that cannot support fibrin formation exhibit compromised antimicrobial host defense. Blood. 2015;126(17):2047-58.

30. Korte $\mathbf{W}$, Riesen W. Latex-enhanced immunoturbidimetry allows D-dimer determination in plasma and serum samples. Clin Chem. 2000;46(6 Pt 1): 871-2.

31. Boisclair MD, Lane DA, Wilde JT, Ireland H, Preston FE, Ofosu FA. A comparative evaluation of assays for markers of activated coagulation and/ or fibrinolysis: thrombin-antithrombin complex, D-dimer and fibrinogen/ fibrin fragment $\mathrm{E}$ antigen. Br J Haematol. 1990;74(4):471-9.

32. Korte W, Riesen WF. Comparability of serum and plasma concentrations of haemostasis activation markers. Clin Chem Lab Med. 2001;39(7):627-30.

\section{Publisher's Note}

Springer Nature remains neutral with regard to jurisdictional claims in published maps and institutional affiliations.

Ready to submit your research? Choose BMC and benefit from:

- fast, convenient online submission

- thorough peer review by experienced researchers in your field

- rapid publication on acceptance

- support for research data, including large and complex data types

- gold Open Access which fosters wider collaboration and increased citations

- maximum visibility for your research: over $100 \mathrm{M}$ website views per year

At BMC, research is always in progress.

Learn more biomedcentral.com/submissions 